\title{
Outcomes of the Expanded Programme on Immunization Pre-Service Training Initiatives in Kenya: A Mixed Methods Study
}

\author{
Iqbal Hossain 1*, Evans Mokaya², Isaac Mugoya ${ }^{2}$, Folake Olayinka1 ${ }^{1}$, Lora Shimp \\ ${ }^{1}$ Maternal and Child Survival Program/JSI, Washington DC, USA \\ ${ }^{2}$ Maternal and Child Survival Program/JSI, Nairobi, Kenya \\ ${ }^{3}$ Immunization Center, JSI, Arlington, VA, USA \\ Email: ^Iqbal_hossain@JSI.com, evansmokaya@gmail.com, Isaac_mugoya@ke.JSI.com, folake_olayinka@JSI.com, \\ lora_shimp@JSI.com
}

How to cite this paper: Hossain, I., Mokaya, E., Mugoya, I., Olayinka, F. and Shimp, L. (2019) Outcomes of the Expanded Programme on Immunization Pre-Service Training Initiatives in Kenya: A Mixed Methods Study. World Journal of Vaccines, 9, 85-98.

https://doi.org/10.4236/wjv.2019.94006

Received: July 29, 2019

Accepted: August 31, 2019

Published: September 3, 2019

Copyright (c) 2019 by author(s) and Scientific Research Publishing Inc. This work is licensed under the Creative Commons Attribution International License (CC BY 4.0).

http://creativecommons.org/licenses/by/4.0/

\begin{abstract}
Background: The Maternal and Child Survival Program of United States Agency for International Development conducted a study in 2017 to assess the outcome of an initiative to strengthen Expanded Programme on Immunization (EPI) pre-service training. The pre-service training initiative was undertaken by the Ministry of Health $(\mathrm{MOH})$ with support from partners in 2012-2016. The overall objective of the study was to assess the adoption and effectiveness of the initiative in the competency (knowledge, skills and attitude) of graduate nurses. Methods: The study included a conveniently selected sample of 14 pre-service training institutions, 23 field practicum sites, and 29 health facilities in western Kenya, and used quantitative and qualitative methods of data collection. Results: All pre-service training institutions were found to have adapted the WHO EPI prototype curriculum. Overall, tutors followed training method in the classroom as suggested in the curriculum, except evaluation of students' learning lacked tests or quizzes. Students had opportunities for hands-on practical experience in the field practicum sites. Graduate nurses were found to have acquired the skills for vaccinating children. However, some pre-service training institutions lacked functional skills labs for practical learning of students. In addition, students did not receive up-to-date information on EPI program, and lacked knowledge and skills on monitoring and documentation of EPI coverage during preservice training. Conclusions: It appears that the EPI pre-service training strengthening initiatives facilitated competency-based EPI training of nurses in Kenya. However, preservice training institutions still have scope for improve-
\end{abstract}


ment in the skills lab, hand-washing practice, providing up-to-date information, and training students on coverage monitoring and documentation.

\section{Keywords}

Pre-Service Training, Graduate Nurses, Immunization, Competency

\section{Introduction}

As of 2015, Kenya had 148 health training institutions, of which 102 are for nursing training, distributed in 36 of 47 counties [1]. The World Health Organization (WHO) recommended in its 2013 guidelines that health professionals training institutions should consider designing and implementing continuous training development programs for faculty and teaching staff that are relevant to the evolving healthcare needs of their countries [2]. However, recent advancements in health care science and technologies were not always incorporated into the pre-service training curricula [3]. In 2011, the WHO Africa Regional Office developed the Expanded Program on Immunization (EPI) prototype pre-service training curriculum (updated in 2015) for competency-based training in nursing and midwifery schools [4].

From 2012-2016, the Kenya Ministry of Health (MOH), in collaboration with the Nursing Council and with the support of EPI partners, launched an initiative to strengthen EPI pre-service training for nurses. The initiative included adaptation of the WHO prototype curriculum and mid-level manager training for nurse tutors from all pre-service training institutions (public, private, and faithbased) across the country. The initiative provided tutors with the WHO EPI prototype curriculum and updated immunization manuals, and advocated to increase the quality and duration of field practicum from 2 weeks to 8 weeks. The Maternal and Child Survival Program (MCSP), the United States Agency for International Development's (USAID) flagship global program that supported the initiative, conducted a study in March-April 2017 to measure the outcome of the initiative in terms of the development of competency (knowledge, skills, and attitudes) among the nursing students. The study was guided by the following research questions:

- To what extent has, the pre-service training institutions improved the immunization teaching and training tools.

- Are graduate nurses competent in providing immunization services once posted to health facilities after completing their pre-service education?

- What are the perceptions of graduate nurses of the EPI training they received during their pre-service education?

- What opinion facility managers and sub-county EPI supervisors have of new graduate nurses competency (knowledge, skills, and attitudes) on immunization? 


\section{Methods and Materials}

\subsection{Study Design}

The study was a mixed-methods cross-sectional assessment. The design enabled the team to examine the outcomes of the interventions by collecting and triangulating qualitative and quantitative data from the training institutions, practicum sites, and health facilities where graduate nurses were posted; the perception of graduate nurse on pre-service training; and the perception of facility managers and sub-county supervisors on the competency of graduate nurses (Figure 1).

\subsection{Selection of Study Sites}

The study was conducted in western Kenya where MCSP carried out its project activities. The sites included in the study were pre-service training institutions, field practicum sites, and health facilities where graduate nurses were posted in Kisumu, Migori, Kakamega, Kisii, Homa Bay, Bomet, Bungoma, Transzoia, Elgeyo Marakwet, and Uasin Gishu counties. The sites for the study were conveniently selected based on whether training institutions were teaching EPI topics during the study period; students were available at the field practicum sites; and data collectors could reach and observe immunization sessions in the health facilities during the data collection period. The selected health facilities were included if a nurse was posted there in 2016 after graduating from pre-service education.

The total study sample included 14 training institutions including faith-based, for-profit, and public (Kenya Medical Training College or KMTC) training institutions, 23 field practicum sites, and 29 health facilities (Table 1).

\subsection{Study Population}

The study population comprised 14 nurse tutors at pre-service training institutions, 28 graduate nurses at health facilities, 21 health facility managers, and 16 sub-county EPI supervisors (Table 2).
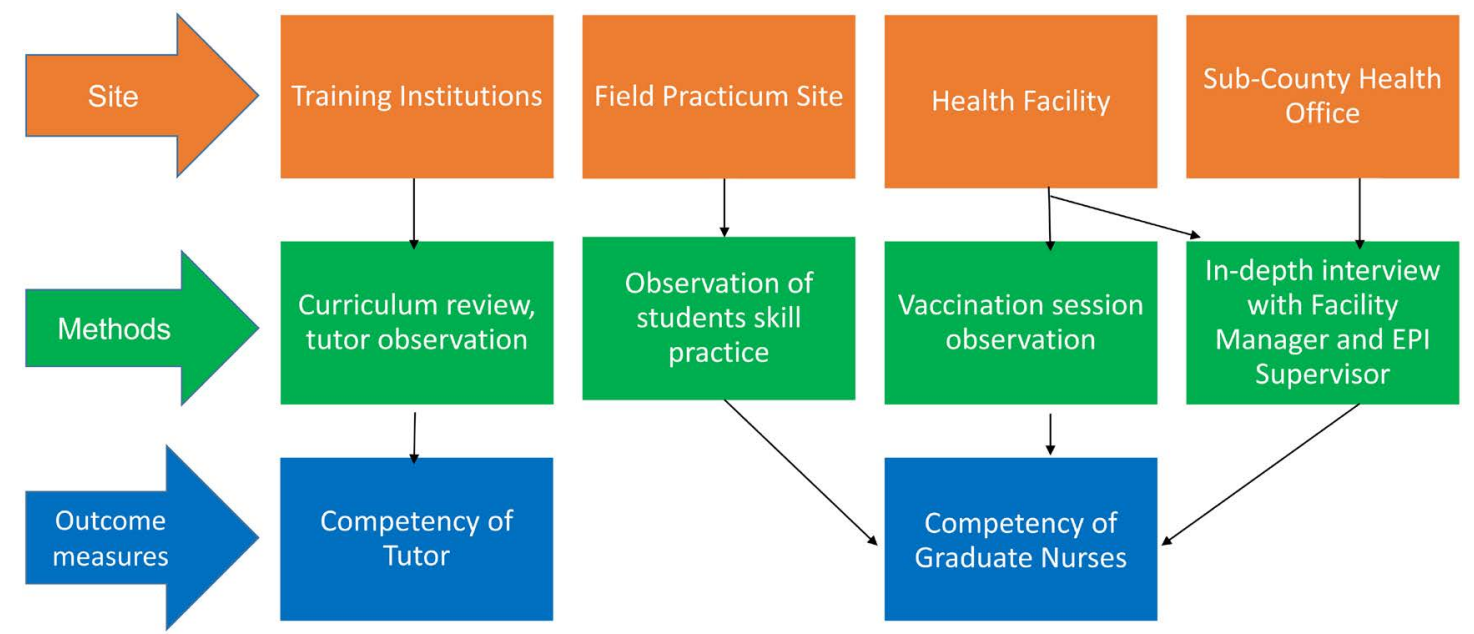

Figure 1. Study design—sites, methods, and outcome measures. 
Table 1. Number and ownership of participating pre-service training institutions, field placement sites, and health facilities in the study, by county.

\begin{tabular}{cccccc}
\hline \multirow{2}{*}{ County } & \multicolumn{2}{c}{ Pre-service training institutions } & Field & $\begin{array}{c}\text { Health } \\
\text { facilities }\end{array}$ \\
\cline { 2 - 4 } Migori & 1 & 0 & 0 & 1 & 1 \\
Kublic (KMTC) & Faith-based & Private & placement sites & 2 & 4 \\
Kakamega & 1 & 0 & 1 & 4 & 5 \\
Kisi & 1 & 0 & 1 & 3 & 5 \\
Homa Bay & 1 & 1 & 0 & 4 & 4 \\
Bomet & 1 & 0 & 1 & 4 & 4 \\
Bungoma & 0 & 2 & 0 & 2 & 2 \\
Transzoia & 1 & 0 & 0 & 1 & 0 \\
Elgeyo Marakwet & 1 & 0 & 0 & 1 & 0 \\
Uasin Gishu & 0 & 0 & 1 & 1 & 5 \\
Total & $\mathbf{7}$ & 0 & 0 & $\mathbf{2 3}$ & $\mathbf{2 9}$ \\
\hline
\end{tabular}

Table 2. Number of tutors, graduate nurses, facility manager, and Sub-county EPI supervisors participated in the study, by county.

\begin{tabular}{ccccc}
\hline & \multicolumn{5}{c}{ Study Participants } \\
\cline { 2 - 5 } County & Nurse Tutors & Graduate Nurses & Facility Managers & $\begin{array}{c}\text { Sub-county EPI } \\
\text { Supervisors }\end{array}$ \\
\hline Migori & 1 & 2 & 1 & 1 \\
Kisumu & 2 & 4 & 4 & 2 \\
Kakamega & 2 & 3 & 5 & 3 \\
Kisi & 2 & 5 & 2 & 2 \\
Hombay & 2 & 4 & 2 & 2 \\
Bomet & 2 & 4 & 1 & 1 \\
Bungoma & 1 & 2 & 2 & 0 \\
Transzoia & 1 & 0 & 0 & 1 \\
Elgeyo Marakwet & 1 & 0 & 0 & 2 \\
Uasin Gishu & 0 & 4 & 4 & 16 \\
Total & $\mathbf{1 4}$ & $\mathbf{2 8}$ & 21 & \\
\hline
\end{tabular}

\subsection{Sample Selection and Inclusion/Exclusion Criteria}

Nurse Tutors. Tutors were included in the study for observation in the classroom if they received EPI training between 2012 and 2016, and excluded if they had not received EPI training.

Graduate nurses: Nurses were included in the study if they graduated from the selected pre-service training institutions in 2016, and excluded if they had received any EPI in-service training. 
Facility managers: Managers were included were included in the study if they were officers' in-charge at the health facility where graduate nurses were posted. All facility managers were available for in-depth interview and no one was excluded.

Sub-county EPI supervisors. Supervisors were included if they were the EPI focal person at the sub-county level, responsible for supervising EPI work in the selected health facilities. All Sub-county EPI supervisors were available for in-depth interview and no one was excluded.

The MCSP data collectors visited all selected pre-service training institutions, field practicum sites, and health facilities; and interviewed all nursing graduates, facility managers, and sub-county supervisors who met the criteria.

\subsection{Ethical Review and Confidentiality of Participants}

The research plan was determined to be exempted from human subject oversight by the John Snow Inc. (JSI) Institutional Review Board (IRB) in the United States and the IRB of Maseno University in Kenya.

The MCSP data collectors obtained consent from all study participants before administering the questionnaires. Participants' names were not collected, and the information they provided was kept confidential during data collection, storage, and analysis.

\subsection{Data Collection Tools}

MCSP developed data collection questionnaires, pre-tested them at pre-service training institutions and health facilities that were not included in the study, and made minor changes in the tools based on the pre-test.

\subsection{Data Analysis}

MCSP entered quantitative data and analyzed using Microsoft Excel and generated simple frequency tables; and transcribed qualitative data into an electronic database and analyzed based on emergent themes.

\section{Results}

\subsection{Improvement of Teaching and Training Tools in the Participating Pre-Service Training Institutions}

The degree to which the participating training institutions improved the pre-service training was evaluated by reviewing adaptation of EPI Prototype curriculum, skills lab, availability of reference manual, competency of tutors, and skills practice of students' in the field practicum sites.

\section{1) Adaptation of WHO EPI prototype curriculum}

Almost all pre-service training institutions found to have adapted the WHO EPI prototype curriculum either all or some priority topics recommended in the WHO EPI prototype curriculum. Five of the seven public (KMTC) training institutions adapted all EPI topics in their nursing curriculums. One adapted only 
the priority EPI topics; and the data collector was not able to review the curriculum of the remaining KMTC site, as the person responsible at the institution was not available during the data collection period. Faith-based and private institutions also varied in their degree of adaptation (Table 3).

2) Skills lab in the pre-service training institutions

Six of the seven KMTCs (but none of the faith-based or private training institutions) had a dedicated room used as a skills lab for the students; one institution did not set up a skills lab due to lack of space. KMTC skills labs had limited functional equipment; for example, only one KMTC had a functioning (though old) EPI refrigerator, two had nonfunctioning EPI refrigerators, and three did not have any refrigerators (functioning or non-functioning) in their skills labs. However, all six KMTC skills labs had a vaccine carrier, safety box, and auto-disable syringes. None of the faith-based or private pre-service training institutions had set up skills lab for the students (Table 3).

\section{3) Availability of reference manuals}

Three of the seven KMTCs (but none of the faith-based or private institutions) had a copy of the WHO EPI prototype curriculum available as a tutors' reference manual at the time of the data collectors' visit. None of the pre-service education institutions had a copy of the EPI manual available as a students' reference manual. Probing revealed that tutors received a hard copy of the WHO EPI prototype curriculum and EPI manual (and a flash drive containing both) when they attended the mid-level managers' trainings. However, the tutors did not print out more copies of the materials and when they were transferred away from the institutions; they did not leave the hard copy of the reference manuals at the institution.

4) Competency of tutors in the classroom

Twelve of the 14 tutors (86\%) observed in the classroom were found to explain the learning objectives for the topic to the students. Nearly all tutors (93\%) used the suggested training methods, covered the full contents for the curriculum topics. However, due to lack of skills labs and/or functional equipment in the labs, students did not receive proper demonstration of immunization materials. Only nine of the 14 tutors (64\%) evaluated students' learning using exercises or quizzes in the classroom (Table 4).

Table 3. Improvement in teaching and training tools in the participating pre-service training institutions, by ownership of the institutions.

\begin{tabular}{cccccc}
\hline $\begin{array}{c}\text { Pre-service training } \\
\text { institution }\end{array}$ & \multicolumn{2}{c}{ Adapted WHO Prototype Curriculum } & Skills Lab & $\begin{array}{c}\text { Reference } \\
\text { Manual }\end{array}$ \\
\cline { 2 - 4 } & All topics & Priority topics & Not available & & \\
\hline $\begin{array}{c}\text { Kenya Medical Training } \\
\text { Colleges (KMTCs) }\end{array}$ & $5 / 7(71 \%)$ & $1 / 7(14 \%)$ & $1 / 7(14 \%)$ & $6 / 7(86 \%)$ & $3 / 7(43 \%)$ \\
$\begin{array}{c}\text { Faith-based pre-service } \\
\text { training institutions }\end{array}$ & $1 / 3(33 \%)$ & $2 / 3(67 \%)$ & - & 0 & 0 \\
$\begin{array}{c}\text { Private pre-service } \\
\text { training institutions }\end{array}$ & $2 / 4(50 \%)$ & $1 / 4(25 \%)$ & $1 / 4(25 \%)$ & 0 & 0 \\
\hline
\end{tabular}


Table 4. Competency of nurse tutors observed in the classroom of the pre-service training institutions.

\begin{tabular}{ccc}
\hline Skills/Knowledge of tutors & Number of tutors $(\mathbf{N}=14)$ & Percentage \\
\hline Explained learning objectives & 12 & 86 \\
Used suggested teaching methods & 13 & 93 \\
Covered content for the topic & 13 & 93 \\
Reinforced learning by asking questions & 13 & 93 \\
Evaluated students by learning objectives & 9 & 64 \\
\hline
\end{tabular}

\section{5) Skills practice of students at the field practicum sites}

Overall, all 23 field practicum sites had the prerequisites for providing students with basic skills in immunization, but there were several notable gaps. Twenty-one of the 23 field practicum sites (91\%) had such fundamental prerequisites as accommodation for the students, a qualified maternal and child health $(\mathrm{MCH})$ nurse or clinical officer to guide students, immunization services, EPI cold chain equipment and commodities, and a sufficient number of clients for students to observe and practice vaccination. However, the field practicum sites of private training institutions did not have EPI standard refrigerators, but refrigerators for home use; therefore, students of these institutions did not get the appropriate practical experience on cold chain and vaccine management. In addition, two private sites were deemed unsuitable as field practicum sites because they did not have an EPI-trained staff member available to guide the students. Students at all sites had opportunity for hands-on experience in EPI cold chain and vaccine management (placing, arranging, and removing vaccines in the refrigerator; packing and unpacking the vaccine carrier; and recording the refrigerator temperature). Students in all but one practicum site were observed providing health education to mothers/caregivers. The majority (96\%) of field practicum sites were visited by tutors to oversee the students' progress and practices. However, students were not universally trained in some important related skills, such as tracking defaulters and monitoring coverage (Table 5).

\subsection{Competency of Graduate Nurses in Providing Immunization Services}

\section{1) Attitudes towards caregivers}

All 29 graduate nurses demonstrated a positive attitude towards caregivers (greeting them, building rapport, and treating them with respect) as observed during the immunization session.

2) Infection prevention practice

A little more than half (52\%) of the graduate nurses washed their hands with soap and water or used hand sanitizer before starting the immunization sessions. The other nurses did not wash their hands because their facilities lacked running water and soap or hand sanitizer. The majority of nurses followed the non-touch technique during vaccination and discarded used syringes and needles in a safety 
box immediately, without recapping (Table 6).

Table 5. Observation findings at the field practicum sites.

\begin{tabular}{lcc}
\hline \multicolumn{1}{c}{ Pre-requisites available } & $\begin{array}{c}\text { Number of } \\
\text { placement sites } \\
\text { (N = 23) }\end{array}$ & Percentage \\
\hline Had immunization services available in the site & 21 & 91 \\
Had EPI equipment available in the site & 21 & 91 \\
Students had hands-on experience in the EPI cold room & 23 & 100 \\
Students had hands-on experience in the immunization session & 23 & 100 \\
Students practiced giving health education to caregivers & 22 & 96 \\
Students were advised to be respectful to caregivers & 23 & 100 \\
Students had hand-on experience with defaulter tracking & 15 & 65 \\
Students had hand-on experience with coverage monitoring & 9 & 39 \\
EPI trained staff available in the site to serve as local guide for students & 21 & 91 \\
Local guide signed student log books & 18 & 78 \\
Tutors visited the site periodically to check progress of the students & 22 & 96 \\
\hline
\end{tabular}

Table 6. Observation findings at the immunization session conducted by the graduate nurses in the health facilities.

\begin{tabular}{|c|c|c|}
\hline Skills and attitudes of graduate nurses at immunization session & $\begin{array}{c}\text { Number of } \\
\text { graduated } \\
(\mathrm{N}=29)\end{array}$ & Percentage \\
\hline Greeted caregivers & 29 & 100 \\
\hline Treated caregivers politely & 29 & 100 \\
\hline Washed hands before starting immunization session & 15 & 52 \\
\hline Used conditioned ice pack in the immunization session & 23 & 79 \\
\hline Checked vaccine vial monitor (VVM) before vaccination & 12 & 41 \\
\hline Checked expiry date of vaccines before vaccination & 12 & 41 \\
\hline Checked home-based record to determine due vaccination dose & 27 & 93 \\
\hline Followed recommended sequence in giving vaccines & 27 & 93 \\
\hline Used non-touch technique in giving injectable vaccines & 26 & 90 \\
\hline Used correct diluent for BCG and Measles vaccines & 19 & $100^{*}$ \\
\hline Kept diluents cold & 19 & $100^{*}$ \\
\hline Disposed used syringe into safety box immediately, without recapping & 28 & 97 \\
\hline Explained to caregivers the possible side-effects and remedies & 14 & 48 \\
\hline Told caregivers when to return for next vaccination & 27 & 93 \\
\hline Tallied vaccine in the tally sheet after each vaccination & 25 & 86 \\
\hline Entered vaccination dates in the home-based record & 29 & 100 \\
\hline Entered vaccination dates in the permanent register & 28 & 97 \\
\hline
\end{tabular}

${ }^{*} \mathrm{~N}=19$ nurses had client for BCG and Measles vaccination in the immunization sessions observed. 


\section{3) Immunization practices}

Table 6 also shows that though the graduate nurses used good immunization practices overall, there were gaps in certain practices. More than three-fourths (79\%) of graduate nurses conditioned the ice packs for the vaccine carriers, a recommended practice of packing vaccines. The majority of graduates performed correctly in terms of following the proper vaccination procedures, telling caregivers when to return for the next vaccine, and registering vaccines administered. However, fewer than half $(41 \%)$ of the graduate nurses checked the vaccine vial monitor and expiry dates of the vaccines before administering them and explained the possible side effects of vaccination and their remedies to the mothers/caregivers.

\section{4) Immunization knowledge}

Table 7 shows that nurses' broader knowledge about vaccination was mixed. Most (90\%) knew how to identify the defaulters in the permanent register and could define a missed opportunity for vaccination. Two-thirds could interpret the stages of the vaccine vial monitor. Over half correctly listed contraindications for immunization. However, the nurses did not have the most up-to-date knowledge of the national EPI: for example, only 11 of the 29 (38\%) knew about the tOPV to bOPV switch and less than one-quarter could to calculate the coverage rate and dropout rate.

5) Impression of the pre-service EPI training (graduate nurses)

Most graduate nurses said that they did not get most up-to-date information during pre-service training on new vaccines (e.g., rotavirus vaccine, inactivated polio vaccine, or measles second dose) and new cold chain technology (e.g., fridge tags for cold chain temperature monitoring) that have been introduced in the national EPI program.

\subsection{Impression of Graduate Nurses' Competency (Managers)}

\section{1) Facility managers}

Two-thirds (67\%) of facility managers felt that the graduate nurses had adequate

Table 7. Immunization knowledge of graduate nurses.

\begin{tabular}{lcc}
\hline \multicolumn{1}{c}{ Immunization knowledge } & $\begin{array}{c}\text { Number of graduates } \\
\text { who could correctly } \\
\text { explain }(\mathbf{N}=29)\end{array}$ & Percentage \\
\hline Contraindication of immunization & 15 & 51 \\
Missed opportunity for immunization & 21 & 72 \\
Trivalent OPV to bivalent OPV switch & 11 & 38 \\
Difference between OPV and inactivated polio vaccine (IPV) & 11 & 66 \\
Stages and interpretation of vaccine vial monitor (VVM) & 19 & 28 \\
Estimation of EPI target population & 8 & 21 \\
Calculation of coverage and dropout rates & 6 & 10 \\
Plotting coverage rates in the monitoring chart & 3 & \\
\hline
\end{tabular}


EPI knowledge and skills to vaccinate children. Managers agreed with graduates that the pre-service training did not provide up-to-date information, including new vaccines, contraindications, and fridge tags. They added that graduate nurses frequently lacked the knowledge and skills needed to prepare monthly summary reports and calculate coverage and dropout rates. However, more than half (52\%) of the managers said that graduate nurses were capable of performing EPI activities even without in-service training. Almost all the managers believed that graduate nurses were respectful to caregivers.

2) Sub-county EPI supervisors

Most sub-county supervisors had the impression that graduate nurses acquired basic vaccination skills during their pre-service training, but lacked knowledge on new vaccines, cold chain maintenance, target estimation, coverage and dropout rate calculations, and how to prepare coverage monitoring charts. Seven of 17 sub-county EPI supervisors (41\%) thought that the graduates would be capable of conducting immunization session when posted in the health facilities. Almost all supervisors believed that graduates nurses were respectful to mothers/caregivers.

\section{Discussion}

Health professional trainings must be designed as a competency-based medical education (CBME), not just as means to obtain certification [5]. Thus, students must acquire knowledge, skills, and attitude to apply in the clinical settings [6]. CBME requires an enabling environment and well-prepared teachers [7] [8]. Over the last decade, the EPI has undergone many changes, introducing new vaccines and technologies in the program and developing new strategies and guidelines to achieve high, equitable immunization coverage [9].

1) Training quality

The MCSP study found that most tutors had good knowledge of the EPI related contents and followed the training methods suggested in the WHO EPI prototype curriculum. However, there was gaps in the learning labs for demonstration of EPI materials to students. None of the faith-based and private pre-service training institutions had skills lab for students. Though most public pre-service training institutions had a skills lab, they did not have functional cold chain equipment. These gaps had consequences: MCSP found that one-third of graduate nurses lacked knowledge on vaccine vial monitors, the marker for vaccine quality. In addition, there were gaps in tutors' performance, more than two-third were not using exercise or quizzes to evaluate students' learning progress.

It also appeared that the pre-service training did not provide students with up-to-date information on the new vaccines and cold chain technologies introduced in the national program. Almost two-third of the graduate nurses did not know about the tOPV to bOPV switch (switching from trivalent bivalent oral polio vaccine) that took place in the same year of their graduation. Most preservice training institutions did not have a copy of the MOH's updated EPI ref- 
erence manual for nursing students. As a result, about half of graduate nurses did not have knowledge on all vaccination contraindications. Tutors received a copy of the reference manual along with WHO EPI prototype curriculum during the training, but they did not leave the materials in the pre-service training institution when they were transferred.

\section{2) Skills practice for students}

For optimal learning, students should receive experiential learning while observing, handling health care materials, using equipment, and practicing service delivery in the clinical placement sites [10]. Field practicum sites should be conducive for learning-adequate space, immunization services for observation and practice, appropriate equipment and commodities, trained personnel to serve as local guide and supervisor, and periodic supervision visits from tutors from the training institutes [11] [12]. Certain factors have been shown to be critical for learning, such as a proper clinical environment, cooperation from staff, and large number of clients at the clinical placement sites [13] [14] [15].

The absence of skills labs and/or equipment in some participating pre-service training institutions was mitigated to some extent by the well-organized field practicum sites. MCSP found that the public health practicum sites were wellequipped and the environment was conducive for learning by students. Most practicum sites had immunization services, an EPI cold chain system with equipment and commodities, a client volume sufficient to allow students to observe and get hand-on experience in the vaccination session, and a qualified $\mathrm{MCH}$ nurse or clinical officer was there to guide students. This contributed to the competency among most graduate nurses observed in the vaccination session.

However, the field practicum sites in private pre-service training institutions had several deficiencies, including trained on-site local guides or mentors, EPI standard refrigerators, and low client volume, which limited opportunities for students. Some graduate nurses particularly graduated from private pre-service training institutions complained that they had to compete with each other to practice vaccination in the field practicum sites. In addition, in most field practicum sites student did not get hands-on experience in immunization coverage monitoring and documentation. This training deficiency was reflected in the graduate nurses' skills. For example, fewer than of one-quarter of graduate nurse had knowledge on estimation of target population, calculation of coverage and dropout rate, and only one in 10 graduate nurses had knowledge on how to prepare a coverage monitoring chart. Observations from both facility and sub-county-level supervisors suggested that the new graduate nurses were able to vaccinate children but were deficient in on monitoring and documentation of immunization coverage.

3) Vaccination practices among nurse graduates

Vaccine safety, injection safety, and infection prevention and control are im- 
portant aspects of immunization services and thus are included in the immunization training [16] [17]. The MCSP study found that graduate nurses had good vaccine and injection safety practices in terms of not reusing syringes, following the non-touch technique when giving injectable vaccines, and discarding used syringes and needles in a safety box immediately without recapping. However, a major deficiency was observed in hand-washing practices: almost half of graduate nurses did not wash hand before conducting vaccinations. There was no running water and soap (or hand sanitizer) available in certain health facilities, so it was not certain whether this was a gap in the nurses' practices, a health system problem, or both.

\section{Limitations of the Study}

MCSP purposively selected the pre-service institutions, field practicum sites, and health facilities for this study. Therefore, findings are not generalizable to all pre-service training institutions in Kenya. Data collectors spent sufficient time at data collection sites to enable sufficient time for observations. However, the presence of the data collectors may have introduced observation bias-participants may have changed their behavior while under observation. The study's use of multiple viewpoints (tutors, graduates, and managers/supervisors) served to limit any potential bias by allowing the researchers to triangulate the quality and outcome of EPI pre-service training.

\section{Conclusion}

Considering findings from the reviews and observations at the pre-service training institutions, field practicum sites, health facilities and impression of graduate nurses, facility managers, and sub-county EPI supervisors, we can conclude that EPI pre-service training improved in Kenya and that the graduate nurses acquired vaccination competency during pre-service training, though, more gaps existed in private training institutions. The improvement in EPI pre-service training can be attributed to the pre-service educational strengthening initiatives taken by $\mathrm{MOH}$ with partner support. Private pre-service training institutions should set up skills labs, and functional EPI equipment and materials should be made available for all public, private and faith-based training institutions. Updated EPI manuals should be given for nursing students in all pre-service training institutions. National EPI office should share information regularly with the pre-service training institutions, particularly when changes in policies and strategies occur, or when new vaccines and technologies added into the program. We recommend continuous knowledge update of nurses through on-line training and for enhancement of their skills; supervisors should conduct on-the-job training and demonstrate skills during monthly meetings. We also recommend for continuing medical education for teachers/tutors of the pre-service training institutions. Finally, we believe that the $\mathrm{MOH}$ and pre-service training institutions of other countries may find these study findings beneficial when planning to strengthen 
their own pre-service immunization training.

\section{Acknowledgements}

Authors would like to thank the management of the participating pre-service training institutions, field practicum sites and health facilities for giving access to data collectors in their facilities for observation/data collection. Sincere appreciation to sub county supervisors, health facility in-charges, and graduate nurses for giving time for in-depth interview and providing valuable information.

\section{Funding}

This study was made possible by the generous support of the American people through the United States Agency for International Development (USAID) under the terms of the Cooperative Agreement AID-OAA-A-14-00028. The contents are the responsibility of the authors and do not necessarily reflect the views of USAID or the United States Government.

\section{Conflicts of Interest}

The authors declare no conflicts of interest regarding the publication of this paper.

\section{References}

[1] Kenya Health Workforce Report: The Status of Healthcare Professionals in Kenya 2015.

https://www.taskforce.org/wp-content/uploads/2018/07/KHWF_2017Report_Fullre port_042317-MR-comments.pdf

[2] World Health Organization (WHO) (2013) Transforming and Scaling up Health Professionals' Education and Training: World Health Organization Guidelines 2013.

[3] Frank, J., Chen, L., Bhutta, Z., et al. (2010) Health Professionals for New Century: Transforming Education to Strengthen Health Systems in an Interdependent World, Education of Health Professionals for the 21st Century: A Global Independent Commission. The Lancet, 376, 1923-1958. https://www.thelancet.com https://doi.org/10.1016/S0140-6736(10)61854-5

[4] WHO Regional Office for Africa (2015) EPI Prototype Curriculum for Nursing Schools in the WHO African Region.

https://afro.who.int/publications/expanded-programme-immunization-prototype-c urriculum-nursing-midwifery-schools-who

[5] Kiguli, E., Oluwabunmi, E., Kiguli, S., et al. (2014) Competency Based Medical Education in Two Sub-Saharan African Medical Schools. Advances in Medical Education and Practice, 5, 483-489. https://doi.org/10.2147/AMEP.S68480

[6] Murarneza, C. and Mtshali, G. (2018) Conceptualization of Competency Based Curricula in Pre-Service Nursing and Midwifery Education: A Grounded Theory Approach. Nurse Education in Practice, 28, 175-181.

https://doi.org/10.1016/j.nepr.2017.09.018

[7] Brynildsen, G., Bjork, I.T., Bernsten, K., et al. (2014) Improving the Quality of Nursing Students' Clinical Placements in Nursing Homes: An Evaluation Study. Nurse Education in Practice, 14, 722-728. 
https://doi.org/10.1016/j.nepr.2014.09.004

[8] Fullerton, J., Thompson, J. and Johnson, P. (2013) Competency-Based Education: The Essential Basis of Pre-Service Education for the Professional Midwifery Workforce. Midwifery, 29, 1129-1136. https://doi.org/10.1016/j.midw.2013.07.006

[9] WHO, Global Vaccine Action Plan, 2011-2020. https://www.who.int/immunization/global_vaccine_action_plan/GVAP_doc_2011_ 2020/en

[10] Feldacker, C., Chicumbe, S. and Dgedge, M. (2015) The Effect of Pre-Service Training on Post-Graduation Skills and Knowledge Retention among Mid-Level Health Care Providers in Mozambique. Human Resources for Health, 13, 20. https://doi.org/10.1186/s12960-015-0011-9

[11] Phafolis, S., Christensen, A., Skolink, L., et al. (2018) Student and Preceptor Perceptions of Primary Health Care Clinical Placement during Pre-Service Education: Qualitative Result from a Quasi-Experimental Study. Nurse Education in Practice, 28, 224-230.

[12] Mumbo, M.H. and Kinaro, J. (2015) Assessment of Quality and Relevance of Curriculum Development in Health Training Institutions: A Case Study of Kenya. $\mathrm{Hu}$ man Resources for Health, 13, Article No. 67. https://doi.org/10.1186/s12960-015-0048-9

[13] Appiagyei, A., Kirinya, R. and Gross, J.J. (2014) Informing the Scale-Up of Kenya's Nursing Work Force: A Mixed Method Study of Factors Affecting Pre-Service Training Capacity and Production. Human Resources for Health, 12, Article No. 47. http://www.human-resources-health.com/content/12/1/47 https://doi.org/10.1186/1478-4491-12-47

[14] Ildarabadi, E., Moonaghi, H.H., Heydari, A., et al. (2015) Vaccination Learning Experiences of Nursing Student: A Grounded Theory Study. Journal of Educational Evaluation for Health Professions, 12, 29. https://doi.org/10.3352/jeehp.2015.12.29

[15] Nikula, A., Rapola, A. and Hupli, M. (2009) Factors Strengthening and Weakening Vaccination Competency. International Journal of Nursing Practice, 15, 444-454. https://doi.org/10.1111/j.1440-172X.2009.01781.x

[16] Vorsters, A., Tack, S., Hendrickx, A., et al. (2010) A Summer School on Vaccinology: Responding to Identified Gaps in Pre-Service Immunization Training of Future Health Care Workers. Vaccine, 28, 2053-2059. https://doi.org/10.1016/j.vaccine.2009.12.033

[17] Pelly, L., MacDougall, D. and Halperin, B. (2010) The Vaxed Project: An Assessment of Immunization Education in Canadian Health Professional Programs. BMC Medical Education, 10, Article No. 86.

http://www.biomedcentral.com/1472-6920/10/86

https://doi.org/10.1186/1472-6920-10-86 